\title{
Are mast cells the Trojan horse in HIV-1 infection?
}

\author{
PIOTR WITCZAK, EWA BRZEZIŃSKA-BEASZCZYK
}

Department of Experimental Immunology, Medical University of Lodz, Łódź, Poland

\begin{abstract}
Mast cells are multifunctional, long-lived cells, widely distributed throughout vascularized tissues. Currently, more and more data indicate that mast cells are susceptible to HIV-1 infection at the progenitor stage of their ontogeny. Progenitor mast cells display expression of surface molecule CD4 as well as chemokine receptors, i.e. CCR5 and CXCR4, prerequisite for HIV-1 cell entry. Interestingly, there are some factors, including certain cytokines and HIV-1 proteins, that modify surface molecule expression and, consequently, affect the mast cell susceptibility to HIV-1. HIV-1-infected progenitor mast cells are distributed to various tissues where mature and become HIV-1 resistant. However, mature mast cells retain the virus in latent form which might be reactivated via Toll-like receptor (TLR) stimulation or IgE cross-linking. Thus, mast cells could serve as a reservoir for HIV-1 replication and spread.
\end{abstract} $(A I D S)$.

Key words: mast cells, human immunodeficiency virus (HIV), acquired immunodeficiency syndrome

(Centr Eur J Immunol 2012; 37 (4): 382-386)

According to the World Health Organization there was estimated 34.0 million people worldwide living with human immunodeficiency virus (HIV) at the end of 2010. In the same year 2.7 million people were newly infected and 1.8 million died from acquired immunodeficiency syndrome (AIDS). Although the sustained decline in morbidity as well as mortality level might be optimistic, it should be borne in mind that still more and more people live with HIV. These strongly indicate that HIV pandemic continues to persist and AIDS remains a critically important clinical problem. Unfortunately, no cure or protective vaccine has been yet developed and commonly used antiretroviral therapies only suppress viremia. The main obstacle in HIV eradication is that this virus latently infects number of cell types via variety of mechanisms. Thus, it is essential to thoroughly investigate particular cells in the context of potential involvement in persistent HIV infection.

It is commonly known that CD4+ T cells are the predominant target for HIV-1. However, there is increasing evidence that also other cells might be significant reservoirs for HIV-1 [1, 2]. Recent reports have suggested that also mast cells can be infected by HIV-1 and could serve as a reservoir for HIV-1 replication and spread. These multifunctional, long-lived cells develop from hematopoietic stem cells in the bone marrow, next circulate as immature progenitors, and complete their maturation within tissues. Mast cells are widely distributed throughout virtually all vascularized tissues, but are especially numerous at anatomical sites that are directly exposed to the environment, including the skin, airways, and gastrointestinal tract as well as in genitourinary tract. It should be stressed that HIV-1 usually enters the body via mucosa and submucosa layers, in which mast cells are extremely numerous [3-6]. Moreover, the high viral loads are observed in plasma at all stages of HIV-1 infection [7], hence circulating precursor cells, including mast cell progenitors, might be densely environed by the virus. Therefore, it could be assumed that mast cells are quickly and heavily exposed to HIV during infection.

Early research did not report significant differences in mast cell density in the mucosal and/or submucosal layers of ileum between HIV-infected and healthy individuals [8-10]. Recent studies, however, have increasingly indicated that mast cell number can vary during HIV-1 infection. The increase in the number of mast cells in cervical

Correspondence: Ewa Brzezińska-Błaszczyk, Department of Experimental Immunology, Medical University of Lodz, Pomorska 251, 92-213 Łódź, Poland, phone +48 4267573 06, fax +48 4267573 06, e-mail: ewab@csk.umed.lodz.pl 
lymph nodes of HIV-positive men, as compared with healthy subjects, were observed [11], and a significantly higher mast cell count were found in the chorionic epithelium of the uterine cervix of HIV-infected women than of non-HIV-infected [12]. Also, the gingival tissue of HIV-1positive patients with chronic marginal periodontitis as well as healthy gingiva displayed higher mast cell density in comparison with non-infected counterparts [13, 14]. These findings could suggest that mast cells are directly or indirectly affected by HIV-1 and associated with infection aftermaths, including AIDS progression.

So far, much attention has been paid to molecular basis of HIV-1 cell entry [15]. Initially, HIV-1 envelope glycoprotein gp120 binds to CD4 molecule on target cell surface $[15,16]$. This linkage triggers conformational changes in the conserved core of gp120 enabling sequential interactions with the chemokine receptors, such as CCR5 and/or CXCR4 [15-17]. The involvement of CCR3 in HIV-1 entry was also suggested [18]. The interaction of CD4 molecule with gp120 as well as co-receptor engagement elicit further conformation changes in the HIV-1 envelope leading to gp41 subunit shift from a non-fusogenic to a fusogenic state. These events promote a fusion between viral and cellular membranes and ultimately virus internalization ensues $[15,16]$. The usage of specific co-receptors by HIV-1 is determined by certain regions of gp120 and dictates virus cell tropism. Hence, R5-tropic (M-tropic) and X4-tropic (T-tropic) HIV-1 strains infect CD4 ${ }^{+}$target cells expressing CCR5 or CXCR4, respectively, whereas dualtropic R5X4 strain can use both CCR5 and CXCR4 co-receptors [17, 18].

The groundbreaking discovery of HIV-1-susceptible cells in the peripheral blood of allergic individuals that exhibit both mast cell and basophil features has shed light on direct mast cell involvement in HIV-1 infection [20]. It was reported that in vivo- as well as in vitro-differentiated mast cell/basophil line expressed surface CD4, CXCR4, CCR3 and CCR5, i.e. molecules prerequisite for HIV-1 entry and, indeed, was susceptible to M-tropic strain of HIV-1. Interestingly, despite the considerable expression of CD4 and CXCR4 molecules mast cell/basophil line were not susceptible to T-tropic strain of HIV-1. According to authors, it is possible that CD4 preferentially associates with CCR5 molecule rather than CXCR4 on the surface of mast cell/basophil cells, that CXCR4 may simply not be functional as a co-receptor for HIV-1 entry or its surface density is inadequate for the effective infection. It should be pointed out that patients with AIDS had HIV-1-infected mast cells/basophils in their peripheral blood [20]. Likewise, human progenitor mast cells differentiated in vitro from cord blood mononuclear cells (CBMCs) expressed surface CD4, CXCR4, CCR5, CCR3 and were susceptible to M-tropic and dualtropic, but not T-tropic, HIV-1 strains [21]. Moreover, CCR5 was shown to be the principal coreceptor utilized by HIV-1 M-tropic isolates to infect progenitor mast cells. The CCR5-specific inhibitor significantly reduced the infection whereas blocking the CCR3 or CXCR4 molecules only slightly decreased virus entry or have no effect, respectively. In contrast, Taub et al. [22] reported that $\mathrm{CD}^{+}, \mathrm{CXCR}^{+}$and $\mathrm{CCR}^{-}$human mast cell (HMC)-1 line was susceptible to X4- but not R5-tropic strain, while $\mathrm{CD}^{+}, \mathrm{CXCR}^{+}$and $\mathrm{CCR}^{+}$primary mast cells were productively infected by either X4- and R5-tropic viruses. Interestingly, surface expression of CD4 molecule for both investigated mast cell populations was very low. Nonetheless, the monoclonal antibodies to CXCR4 or CD4 partially blocked HMC-1 infection by X4-tropic strain and the use of both antibodies completely blocked the infection. Thus, these molecules were demonstrated to be indispensable for X4-tropic HIV-1 entry. Notably, HMC-1 and primary mast cells after 56-day in vitro exposure to stem cell factor (SCF) were capable of being infected by HIV-1. Other studies by Sundstrom et al. [23, 24] provided evidence that in vivo differentiated $\mathrm{CD} 4^{+}, \mathrm{CXCR} 4^{+}$and $\mathrm{CCR} 5^{+}$progenitor mast cells, developed from adult circulating CD34 ${ }^{+}$ pluripotent progenitors, were significantly susceptible to HIV-1 R5-tropic strain and marginally to X4-tropic strain, and progenitor mast cells derived in vitro from $\mathrm{CD} 34^{+}$cord blood stem cells - to R5-tropic strain. In addition, HIV-1 infected progenitor mast cells were present in the circulation of HIV-1-infected pregnant women [23].

Recent studies have also indicated that during maturation mast cells lose CD4 molecules and co-receptors utilized by HIV-1 for cell entry and become resistant to infection [23]. In vitro-differentiated from CBMCs human mature mast cells expressed CCR3 and some CXCR4 but not CCR5 or CD4 and did not indicate susceptibility to either M-tropic and T-tropic HIV-1 strains [21]. Similarly, mature mast cells developed in vitro from circulating peripheral blood mononuclear cells (PBMCs) as well as mast cells isolated from placental tissues were highly resistant to infection with X4- and R5-tropic strains of HIV-1 due to lack of CCR5, CXCR4 and CD4 molecules [23].

It is important to know that some factors affect the expression of certain surface receptors utilized by HIV-1 for cell entry thus altering mast cell susceptibility to HIV-1. Transforming growth factor (TGF)- $\beta 1$ and tumor necrosis factor (TNF), cytokines overexpressed in AIDS individuals, upregulate surface expression of CCR5 and CD4 on HMC-1 as well as CCR5 and CXCR4 on primary mast cells [22]. HMC-1 preincubated with TGF- $\beta 1$ or TNF are more susceptible to X4- and R5-tropic viruses. In contrast, cord blood-derived mast cells/basophils exposed to interleukin (IL)-16, that interacts with CD4 molecule, show decreased susceptibility to M-tropic HIV-1 infection [25]. HIV-1 Tat protein, secreted by HIV-1-infected cells to support viral gene expression and replication, was demonstrated to upregulate CCR3 expression on FceRI+ cells, thus may enhance susceptibility to subset of HIV-1 viruses that use this receptor for cell entry [26]. The research by Sundstrom et al. [27] indicated that $\operatorname{IgE}$ treatment of progenitor mast cells derived 
from human $\mathrm{CD} 4^{+}$cells significantly and uniquely enhances mRNA as well as surface expression of CXCR4, but not CCR3 or CCR5, what correlates with an increased susceptibility of these cells to HIV-1 X4-tropic strain. Specific or nonspecific cross-linking of IgE bound to FceRI induces CXCR4 mRNA expression and susceptibility to $\mathrm{X} 4$-tropic virus as well, however much more rapidly than progenitor mast cell treatment with IgE alone. Notably, increase of CXCR4 expression levels and vulnerability to $\mathrm{X} 4$-tropic strain are dependent on progenitor mast cell maturational stage.

These data indicated that mast cells are transiently susceptible to HIV-1 during their ontogeny. Circulating progenitor mast cells that express surface molecules of HIV-1 entry are distributed to different tissues, where mature and, due to loss of this expression, become HIV-1 resistant. Intriguing is whether HIV-1 can establish latent form within these cells and preserve until reactivation. If does, it could be hypothesized that mast cells may constitute a long-term shelter for transcriptionally silent HIV-1. Hence, it has been important to determine HIV-1 ability to replicate within mast cells as well as presence of integrated HIV-1 DNA sequences in genome of mature mast cells. No less significant is the search for factors that could initiate productive HIV-1 reactivation in these cells. HIV-1 persistence is upheld either in different anatomical sections or in viral reservoirs of latently infected cells that contain proviral genes [28]. Cells harboring HIV-1 integrated DNA must be capable of maintaining the virus in transcriptionally silent as well as replication-competent form that, when reactivated, infects another target cells. Additionally, viral reservoir must be able to survive the infection and manifests sufficient longevity in order to restore the population of infected cells eliminated by the host immune response.

First, it was demonstrated that HIV-1 is able to productively infect mast cell/basophil line obtained in vivo as well as in vitro [20]. Detectable levels of HIV-1 DNA as well as p24 viral antigen were visible in conditioned medium in 12 day postinfection for mast cells differentiated in vivo. Mast cells obtained in vitro displayed even higher amount of retrovirus and p24 antigen occurred already in 3 day after HIV-1 treatment. Also Bannert et al. [21] reported that HIV-1 can replicate in mast cells differentiated in vitro. HIV-1-exposed progenitor mast cells showed the progressive augmentation in p24 antigen levels in the culture supernatant up to 21 day postinfection. More importantly, the authors provided evidence that after maturation HIV-1infected mast cells become resistant to the virus that preserved its replicative capacity. Other authors reported, by determining the p24 antigen in the cell-free culture supernatants, that HIV-1 can replicate in HMC-1. Interestingly, SCF treatment did modestly increase viral p24 levels on day 56 on the HMC-1 [22]. These findings have laid the groundwork of a model for mast cells as long-lived sanctuary for persistent HIV infection. This concept was reinforced by
Sundstrom et al. [24] who investigated HIV-1 replication kinetics in in vitro-maturating mast cells. HIV-1 p24 antigen levels were readily detectable by day 7 postinfection, peaked at 2-3 week as HIV-1-resistant mature mast cells emerged, and then steadily declined to below detectable limits by 10 week - at which point HIV-1 proviral DNA was confirmed by PCR. Moreover, virus replicative capacity preserved in both HIV-1 infected progenitor and latently infected mature mast cells. Moreover, mast cell stimulation via Toll-like receptor (TLR)-2, TLR-4, and TLR-9 significantly enhanced viral replication without promoting degranulation, apoptosis and cellular proliferation. The discovery of these biologically relevant signals enhancing HIV1 production in mature mast cells resistant to virus-mediated cytopathology led the authors to speculate that mast cells are potentially more steady, durable and virulent than other cell viral sanctuaries. In their subsequent studies placental tissue mast cells isolated from HIV-1-infected women on active antiretroviral therapy (HAART) during pregnancy were shown to be long-lived, resistant to HIV-1 and encompass replication-competent integrated HIV-1 proviral DNA. Moreover, HIV-1-infected prMCs, from which placental mast cells evolve, were cultured in vitro with mast cell growth factors. Although 9 week-old mast cells were still productively infected as detectable p24 antigen levels were observed, by 12 week of culture the infection became latent due to negative results of p24 molecule or mRNA transcript measurements by ELISA or PCR, respectively. Reactivation of latently infected mature mast cells, however, induced a dose-dependent response of viral replication and production of infectious virus after agonist stimulation of TLR-2, TLR-4, TLR-9 or IgE cross-linking [23]. In contrast, Nelson et al. [29] did not determine active HIV1 replication in tissue mast cells from HIV-1-infected patients. The comparative immunohistochemical analysis of tissues obtained from different anatomic sites, i.e. lymph nodes, cervix, parotid glands, nasopharynx, and gastrointestinal tract, of 10 donors showed no distinct co-localization between mast cell tryptase and HIV-1 p24 antigen expressing cells. Importantly, the same analysis for macrophages indicated co-location or proximity to p24 antigen expressing cells. However, these findings may contradict the hypothesis of mast cells as an important HIV-1 reservoir, further studies aimed at determining whether integrated viral gene sequences are present in these cells are necessary.

It has been difficult to eradicate HIV-1 in humans in part because of virus capacity to infect long-lived cells. Certainly, ability to establish reservoir in long-lived cells hampers HIV-1 eradication in humans [30]. It should be stressed that, out of the non-T cell lineages that can be infected with HIV-1, progenitor mast cells are quaint in that they are recruited to diverse tissue compartments in response to tissue growth, remodeling, injury or inflammation $[4,5]$. HIV-1 may utilize progenitor mast cells for effective viri- 
on distribution to infect cells within different tissue compartments, otherwise difficult to access or even unreachable for the virus. Conceivably, HIV-1-infected progenitor mast cells or mature mast cells can infiltrate lymphoid organs and escalate transfer of the virus to $\mathrm{CD}^{+} \mathrm{T}$ cells. In fact, mast cells migrate to local lymph nodes under some inflammatory conditions [31] and cervical lymph nodes of AIDS individuals show augmented numbers of mast cells [10]. It should be also stressed that progenitor mast cells can extravasate to central nervous system via blood-brain barrier where, as mature mast cells, transport the virus to HIV1-susceptible microglia or astrocytes [32]. Importantly, mucosal or submucosal mast cell number in biopsies of HIV-1-infected individuals is usually increased or unimpaired, thus these cells might be a significant source of viral production at these tissues. Interestingly, Sundstrom et al. [23] speculated on the potential role of mast cell reservoir in mother-to-child transmission of HIV-1 infection.

Taken together, we still have insufficient knowledge to determine the significance of mast cells in HIV-1 infection. Determining the cell populations that constitute shelter for HIV-1 as well as the manner in which reservoirs are established have been a high priority in the elaboration of effective antiretroviral therapies $[33,34]$. Mast cells may provide an important and useful model for investigation how latency originates and what are the connections between cell development and susceptibility to latent and productive infections.

This work was supported by grant from the Medical University of Lodz (grant 502-03/6-164-01/502-64-005).

The authors declare no conflict of interest.

\section{References}

1. McNamara LA, Collins KL (2011): Hematopoietic stem/precursor cells as HIV reservoirs. Curr Opin HIV AIDS 6: 43-48.

2. Alexaki A, Liu Y, Wigdahl B (2008): Cellular reservoirs of HIV-1 and their role in viral persistence. Curr HIV Res 6: 388400.

3. Gurish MF, Boyce JA (2006): Mast cells: ontogeny, homing, and recruitment of a unique innate effector cell. J Allergy Clin Immunol 117: 1285-1291.

4. Okayama Y, Kawakami T (2006): Development, migration, and survival of mast cells. Immunol Res 34: 97-115.

5. Metcalfe DD, Baram D, Mekori YA (1997): Mast cells. Physiol Rev 77: 1033-1079.

6. Górska A, Niedoszytko M, Lange M (2011): Mastocytosis and clonal mast cell activation syndrome. Post Dermatol Alergol 28: 217-223.

7. Piatak M Jr, Saag MS, Yang LC, et al. (1993): High levels of HIV-1 in plasma during all stages of infection determined by competitive PCR. Science 259: 1749-1754.

8. Irani AM, Craig SS, DeBlois G, et al. (1987): Deficiency of the tryptase-positive, chymase-negative mast cell type in gastrointestinal mucosa of patients with defective T lymphocyte function. J Immunol 138: 4381-4386.
9. Bishop PE, McMillan A, Gilmour HM (1987): Immunological study of the rectal mucosa of men with and without human immunodeficiency virus infection. Gut 28: 1619-1624.

10. Cummins AG, LaBrooy JT, Stanley DP, et al. (1990): Quantitative histological study of enteropathy associated with HIV infection. Gut 31: 317-321.

11. Paiva DD, Morais JC, Pilotto J, et al. (1996): Spectrum of morphologic changes of lymph nodes in HIV infection. Mem Inst Oswaldo Cruz 91: 371-379.

12. Guimarães JV, Costa FB, Andrade WM, et al. (2011): Quantification of mast cells in the uterine cervix of women infected with human immunodeficiency virus. Ann Diagn Pathol 15: 318-322.

13. Myint M, Steinsvoll S, Yuan ZN, et al. (2002): Highly increased numbers of leukocytes in inflamed gingiva from patients with HIV infection. AIDS 16: 235-243.

14. Naesse EP, Schreurs O, Helgeland K, et al. (2003): Matrix metalloproteinases and their inhibitors in gingival mast cells in persons with and without human immunodeficiency virus infection. J Periodontal Res 38: 575-582.

15. Pierson TC, Doms RW (2003): HIV-1 entry and its inhibition. Curr Top Microbiol Immunol 281: 1-27.

16. Kwong PD, Wyatt R, Robinson J, et al. (1998): Structure of an HIV gp120 envelope glycoprotein in complex with the CD4 receptor and a neutralizing human antibody. Nature 393: 648-659.

17. Berger EA, Murphy PM, Farber JM (1999): Chemokine receptors as HIV-1 coreceptors: roles in viral entry, tropism, and disease. Annu Rev Immunol 17: 657-700.

18. Choe H, Farzan M, Sun Y, et al. (1996): The $\beta$-chemokine receptors CCR3 and CCR5 facilitate infection by primary HIV-1 isolates. Cell 85: 1135-1148.

19. Berger EA, Doms RW, Fenyö EM, et al. (1998): A new classification for HIV-1. Nature 391: 240.

20. Li Y, Li L, Wadley R, et al. (2001): Mast cells/basophils in the peripheral blood of allergic individuals who are HIV-1 susceptible due to their surface expression of CD4 and the chemokine receptors CCR3, CCR5, and CXCR4. Blood 97: 3484-3490.

21. Bannert N, Farzan M, Friend DS, et al. (2001): Human Mast cell progenitors can be infected by macrophagetropic human immunodeficiency virus type 1 and retain virus with maturation in vitro. J Virol 75: 10808-10814.

22. Taub DD, Mikovits JA, Nilsson G, et al. (2004): Alterations in mast cell function and survival following in vitro infection with human immunodeficiency viruses-1 through CXCR4. Cell Immunol 230: 65-80.

23. Sundstrom JB, Ellis JE, Hair GA, et al. (2007): Human tissue mast cells are an inducible reservoir of persistent HIV infection. Blood 109: 5293-5300.

24. Sundstrom JB, Little DM, Villinger F, et al. (2004): Signaling through Toll-like receptors triggers HIV-1 replication in latently infected mast cells. J Immunol 172: 4391-4401.

25. Qi JC, Stevens RL, Wadley R, et al. (2002): IL-16 regulation of human mast cells/basophils and their susceptibility to HIV-1. J Immunol 168: 4127-4134.

26. de Paulis A, De Palma R, Di Gioia L, et al. (2000): Tat protein is an HIV-1-encoded $\beta$-chemokine homolog that promotes migration and up-regulates CCR3 expression on human FceRI+ cells. J Immunol 165: 7171-7179.

27. Sundstrom JB, Hair GA, Ansari AA, et al. (2009): IgE-FceRI interactions determine HIV coreceptor usage and susceptibility to infection during ontogeny of mast cells. J Immunol 182: 6401-6409. 
28. Blankson JN, Persaud D, Siliciano RF (2002): The challenge of viral reservoirs in HIV-1 infection. Annu Rev Med 53: 557-593.

29. Nelson AM, Auerbach A, Man YG (2009): Failure to detect active virus replication in mast cells at various tissue sites of HIV patients by immunohistochemistry. Int J Biol Sci 5: 603-610.

30. Chun TW, Fauci AS (1999): Latent reservoirs of HIV: obstacles to the eradication of virus. Proc Natl Acad Sci U S A 96: 10958-10961.

31. Wang HW, Tedla N, Lloyd AR, et al. (1998): Mast cell activation and migration to lymph nodes during induction of an immune response in mice. J Clin Invest 102: 1617-1626.

32. He J, Chen Y, Farzan M, et al. (1997): CCR3 and CCR5 are co-receptors for HIV-1 infection of microglia. Nature 385: 645-649.

33. Schrager LK, D'Souza MP (1998): Cellular and anatomical reservoirs of HIV-1 in patients receiving potent antiretroviral combination therapy. JAMA 280: 67-71.

34. Pierson T, McArthur J, Siliciano RF (2000): Reservoirs for HIV-1: mechanisms for viral persistence in the presence of antiviral immune responses and antiretroviral therapy. Annu Rev Immunol 18: 665-708. 